\title{
Enabling Vertical Industries Adoption of 5G Technologies: a Cartography of evolving solutions
}

\author{
Anastasios Zafeiropoulos, Panagiotis \\ Gouvas, Eleni Fotopoulou, George \\ Tsiolis, Thanos Xirofotos \\ UBITECH \\ Athens, Greece
}

Stamatia Rizou

Singular Logic S.A.

Athens, Greece

Athul Prasad

Access Research, NOKIA Bell Labs

Espoo, Finland

\author{
Anastasius Gavras, Maria Joao \\ Barros \\ EURESCOM \\ Heidelberg, Germany
}

\author{
Marco Gramaglia \\ Universidad Carlos III de Madrid \\ Madrid, Spain
}

\author{
Mikael Fallgren \\ Ericsson Research \\ Ericsson AB \\ Stockholm, Sweden
}

\author{
Gino Carrozzo \\ Nextworks \\ Pisa, Italy
}

\author{
Xavier Costa-Perez \\ NEC Laboratories Europe \\ Heidelberg, Germany \\ xavier.costa@neclab.eu
}

\author{
Anna Tzanakaki, Dimitra \\ Simeonidou \\ University of Bristol \\ Bristol, UK
}

\author{
Raul Muñoz, Ricard Vilalta \\ Centre Tecnològic de \\ Telecomunicacions de Catalunya \\ Castelldefels (Barcelona), Spain
}

\begin{abstract}
G network technologies are evolving in a tremendous pace, enhancing the potential for being adopted and exploited by vertical industries and serve advance networking requirements needs. Towards this direction, a set of 5G PPP projects are providing contributions for tackling aspects related to the overall lifecycle of 5G vertical applications design, development and deployment, including the activation and management of the appropriate network services. In this paper, a cartography of a set of novel solutions facilitating the adoption of $5 \mathrm{G}$ technologies by vertical industries is presented, aiming at identifying set of challenges and relevant solutions as well as potential synergies among the related projects.
\end{abstract}

Keywords-5G PPP, SDN, NFV, MEC Orchestration, Software Development Kit, Vertical Industries

\section{INTRODUCTION}

$5 \mathrm{G}$ network design and evolution is considered as a key enabler to support the introduction of digital technologies in economic and societal processes, and it is leading to the fourth industrial revolution across multiple sectors, as reported in the "5G empowering vertical industries" whitepaper produced by the 5G-PPP association [1]. The deep integration of verticals with the underlying new generation network is considered as one of the key differentiators between $4 \mathrm{G}$ and $5 \mathrm{G}$ systems to open truly global markets for innovative digital business models. By cementing strong relationships between vendors, operators and verticals, $5 \mathrm{G}$ will open the field to new business value propositions [1]. However, to achieve the proper integration of verticals with the $5 \mathrm{G}$ network, the specific requirements, challenges and Key Performance Indicators (KPI) of the various application scenarios have to be considered. Given that $5 \mathrm{G}$ networks will be the platform enabling growth in many industries, the set of services per vertical industry is going to cater for a diverse set of requirements in terms of provisioning and management of infrastructure resources [2]. Such requirements may span from radio access network requirements to a set of deployment constraints in the edge, transport and core infrastructure. To fulfil the set of identified requirements while taking into account the peculiarities associated with each vertical industry, $5 \mathrm{G}$ networks have to be operated by intelligent orchestration platforms that are able to support end-to-end services provision over programmable infrastructure [3]. By leveraging virtualization and softwarization technologies, developers and operators will better match needs and capabilities, building application-aware networks and network-aware applications. This joint power is considered one of the main drivers of innovations enabled by $5 \mathrm{G}$, as stated at the "View on $5 \mathrm{G}$ Architecture" of the 5G PPP Architecture Working Group [3].

Towards this direction, in all the network parts, there is a transition from today's "network of entities" to a "network of (virtual) functions" approach, where a network service can be dynamically composed on an "on-demand", "on-the-fly" basis [3]. Such a composition has to be realized taking into account requirements denoted on behalf of the vertical industries and facilitated by a service-specific grouping of network functions to logical entities and the mapping of logical to physical 
architecture, which is in full accordance with the envisioned ETSI Network Functions Virtualization (NFV) architectural framework [4].

The term network slice is introduced to serve such a diverse ecosystem. A network slice is a part of the infrastructure (consisting mainly of virtualized resources, virtual/physical network functions and network services) that aims to support a set of services and meet the desired KPIs of the service providers. A network slice is created on demand based on the available network, compute and storage resources and the requirements imposed on behalf of the services provider [5].

Taking into account the aforementioned needs, we propose in this paper a cartography of $5 \mathrm{G}$ vertical industries which are currently influencing the design of $5 \mathrm{G}$ systems and solutions in a group of $\mathrm{R} \& \mathrm{D}$ projects of the $5 \mathrm{G}$ PPP programme (i.e. MATILDA, 5GTANGO, 5GCity, 5G-Xcast, 5G-MoNArch, SLICENET，5G-MEDIA，IoRL，5G-TRANSFORMER，5GPICTURE, 5GCAR, BlueSPACE). The examined solutions tackle the overall lifecycle of $5 \mathrm{G}$ vertical applications design, development and deployment over $5 \mathrm{G}$ networks, including the activation and management of the appropriate network services. Specifically, solutions for the design and development of network applications and network services (e.g. metamodels, software development kits, validation and verification frameworks), solutions supporting resources and network slices creation and management and solutions regarding management and orchestration mechanisms in the various layers (e.g. Network Function Virtualization (NFV), Multi-access Edge Computing (MEC), Software Defined Networking (SDN), vertical applications orchestration) are included in the overall cartography.

\section{CHALLENGES FOR ENABLING VERTICALS TO ADOPT 5G} TECHNOLOGIES

\section{A. Design and Development of Applications and Services}

A crucial factor for enabling verticals to take advantage of $5 \mathrm{G}$ ecosystems relates to the capability to take into consideration network requirements denoted in the vertical applications software during their deployment and execution over a $5 \mathrm{G}$ infrastructure. Such requirements can be denoted based on the design and specification of appropriate applications' description metamodels, leading to set of descriptors accompanying the developed software. These applications can be considered 5G-ready in the sense that, through the development of translation mechanisms between application-specific requirements and programmable infrastructure requirements, optimal deployment and operation can be guaranteed. Specifically, the set of application-defined requirements along with their prioritization can lead to the instantiation of the appropriate network slice.

On the network operators' side, a similar challenge relates to the design and development of virtual network functions and network services that can be verified and validated for being used for serving a set of network requirements, as identified by the vertical industries. Towards this direction, the specification of metamodels and the design and implementation of development kits as well as validation and verification frameworks targeted to network services is necessary. In both cases, the specification and exposure of open APIs for accessing the various development, validation and verification kits, as well as experimentation based on the available software, is crucial. Through open APIs, a rich set of primitive functions for network and vertical application layer services can be made available, leading to composition of various network services required by the applications, as well as network-aware vertical applications. The developed software can support SDN, NFV and MEC use cases, as well as use cases associated with blending of such technologies for meeting the imposed requirements from the various vertical domains (e.g. automotive, media applications, emergency communications, IoT).

\section{B. Resources/Network Slices Management}

With regards to the creation and management of network slices targeted to requirements per vertical applications or cluster of applications, a set of challenges are identified. For supporting integration of vertical applications, there is a need for the design and implementation of mechanisms able to translate a slice intent to the appropriate network slice template. A slice intent corresponds to the set of network requirements -in terms of functionalities and deployment constraints- that have to be fulfilled during the network slice instantiation process. The translation involves the appropriate mapping of the provided intent to a network slice template offered by a network operator. This mapping has to be compatible with different resource and slice management, potentially using advanced QoS management frameworks [6].

For example, the multicast / broadcast of automotive data would require dedicated slices, with pre-defined SLAs in terms of transport network resources and related requirements. Similar constraints would be applicable to verticals requiring broadcast of reliability and latency constrained traffic with high data rate requirements, such as VR. For such traffic, the need to reserve edge cloud resources for possible caching needs to be considered while setting up the network slice. For massive IoT traffic such as device updates which has limited reliability requirements, the data could be delivered using a best-effort slice [7]. The slice could also be managed depending on the delivery type. For example, a public safety slice could be used for broadcast data, which essentially does not require uplink resources.

Following this mapping, a set of challenges with regards to the management of a network slice, includes: (i) the ability to decompose a NS instantiation request to the required network resources, as well as to collect and process information about the running NSs, (ii) the ability to dynamically allocate or deallocate resources (NW slices/shares and MEC nodes) to the Service Providers according to their own SLA (QoS, etc.); (iii) the ability to scale and dynamically reallocate resources to the Service Providers to align with the changing needs of different users and applications, as well as achieve graceful degradation when resources are temporarily unavailable; (iv) the ability to shorten creation times to support just-in-time deployment of virtualized applications (e.g., in 100 of msecs or less) and migration times (down to 100 msecs or less); (v) the provision of isolation and security functionalities among the supported 
slices/shares/MEC nodes (multitenancy) and (vi) the support of resiliency mechanisms for maintaining normal network operation even during and after network malfunctions.

\section{Management and Orchestration}

Given the creation of a network slice targeted to a set of applications with specific network requirements, management and orchestration mechanisms are applied in various layers, namely applications/MEC orchestration, NFV/SDN orchestration, transport xhaul resource orchestration, OSS/BSS systems and network slices management. A set of orchestration challenges exists for the supported mechanisms per layer, while a major challenge is posed by the need for interoperability and separation of concerns among the orchestration mechanisms across layers. Given the development of various solutions per layer, the specification and standardization of open interfaces is important. Through standardized interfaces, application deployment requests can be provided by vertical industries' stakeholders towards the network operators' slice management (or OSS/BSS) systems. Subsequently, it is the role of network function orchestration mechanisms on behalf of the operator to facilitate the activation and management of the required network services. Additionally, VNFs need to coexist with more traditional PNFs, while NFV network services need to be orchestrated considering jointly both type of network functions, i.e. PNFs and VNFs, each of them with their specific constraints, lifecycles, configuration and monitoring options.

With regards to slice management, it is also required to design orchestration abstractions that simplify the orchestration of sub-slices deployed across multiple administrative domains to establish a single E2E slice. By establishing these abstractions, which can hide the implementation of a given service, it is possible for a single network operator to effectively manage a service that is built of components owned by other network operators with limited functional impact.

A key challenge is also regarding the availability of intelligent orchestration mechanisms capable to implement the lifecycle management and orchestration of 5G-based services, including edge network and computing services (e.g. media applications with strict QoS demands). Such challenges are concerned with both vertical application orchestration mechanisms and network services orchestration mechanisms. The first ones are responsible for providing the appropriate slice intent, including all the edge computing requirements as well as layer 4-7 functionalities for optimally serving edge computing applications, while the latter ones are responsible for managing the required network services and functions (e.g. mobility, live migration of virtual network functions). Dynamic horizontal and vertical scaling capabilities, support of autonomic and reactive orchestration mechanisms, optimal deployment and runtime policies enforcement mechanisms as well as provision of secure placement and management mechanisms must be provided.

\section{SOFTWARE DEVELOPMENT AND VALIDATION KITS}

A series of development, validation and verification environments are developed within existing 5G PPP projects, focusing mainly on the design and development of vertical applications, the design and development of virtual network functions and network services or both.

In MATILDA, a development environment for applications denoted in the form of an application graph as well as VNFs and NSs is provided. Each application graph consists of a set of application components along with a set of graph links between required and exposed component interfaces. Networking requirements of the application graph -representing the vertical application- are made available through a descriptor, leading to the selection of the appropriate network slice. Design of applications and network services is facilitated through a graph composer, while the developed software is validated based on a set of defined metamodels.

5GCity advocates a more data-centric programming model based on Flow Based Programming (FBP) [8], in which functional components can be assembled into applications by connecting them using pipes. Under this model, applications can adapt by rewiring components, adding/removing functions or changing their implementation. 5GCity is extending the FBP model by using smart-pipes, i.e. allowing in-network functions such as aggregation and filtering. This can allow edge services to adapt to resource availability, handle failures, and network configuration changes (for example, node migration).

The 5GTANGO SDK aims at providing the developer with a powerful set of tools to develop, test and evaluate NFV-based Network Services. The resulting toolset builds further on the ecosystem of SONATA SDK, and further evolves it, given the changing state-of-the-art, modified programming model and Service Platform. 5GTANGO develops also a Validation and Verification (V\&V) framework aiming at validating and verifying appropriate operation of VNFs and NSs by ensuring that they pass a range of tests and thus meet a core set of requirements. The produced software is made available for adoption and usage by instantiations of the 5GTANGO Service Platform by Service Providers.

The 5G-MEDIA approach relies on an Application/Service Development Kit (5G-MEDIA SDK) that assists the function, application and service development, emulation, testing and validation process. More specifically, the 5G-MEDIA SDK tools allow to define Media Service forwarding graphs, to prove and package the various functions as well as to emulate behaviours of the virtualized infrastructure, to accelerate application development and provide a testing environment to be utilized prior to service deployment. The 5G-MEDIA SDK tools enable also the use of the innovative concept of the Function as a Service (FaaS) approach, where developers do not care about the low-level details related to the virtual computing and storage infrastructure, thus drastically contributing to reduce the service creation time cycle and maintenance effort. In this line, the service developers will be able to create the so-called FaaS VNFs, i.e., VNFs that are instantiated upon the detection of specific events.

\section{Network SLice CREATION AND MANAGEMENT}

Management and orchestration of slices is also triggered by a set of projects by the need to map the configuration of a 
network slice with the QoS requirements imposed through the vertical applications.

SLICENET has defined technical use cases that detail the step necessary to perform several operations in the scope of slice creation and lifecycle management. To accommodate slice management functions towards vertical applications and operations, a one-stop API has been defined and partitioned into two management spaces. A vertical one will be utilized by vertical applications and use cases and a horizontal one will be utilized by administrative domain operations. The difference between the two spaces relates with the roles assigned to the users. Each Network Function is exposed through two different views, listing the technical details that need to be considered for combining a NF with other NFs, as well as qualitative details that can be used to influence the features of any higherlevel synthesis an NF is related. The One-Stop API, as used by verticals is based on the provisioning of a marketplace populated with NS Templates. Service selection is provided along with a number of configurable parameters that are exposed by NS and NSS templates. Thus, the customer is able to define location, performance, QoS/QoE and scaling parameters for the customization of the ordered service.

5GTANGO is going to support flexible mechanisms for orchestrating and lifecycle management of network slices aligned with 3GPP activities-, taking into account vertical application requirements and defined SLAs. Complex resource allocation schemas, fitted into dedicated network slice blueprints suitable for vertical industries are going to be supported, along with novel isolation and customizable features, E2E telemetry and monitoring, and E2E orchestration. Novel slice operational features such as slice elasticity, and scalable slice resource scheduling will be introduced. Within the 5GTANGO Network Slice Manager, one can identify two main components: a) the Slice Lifecycle Manager, which is responsible for assigning services and applications to network slices and for managing the lifecycle of these slices; and b) the Slice2NS Mapper, which is responsible for mapping network slices to NFV Network Services (NS).

In 5G-Xcast, slices are proposed to be adapted depending on the traffic type, while in MATILDA the slice intent is going to be created taking into account the network requirements of a vertical applications (as denoted within the relevant descriptor). In 5G-Xcast, the main idea is that based on the SLAs and requirements of the traffic type, the network slice manager [9][10] could interact with a service capability exposure function, access and mobility management function (AMF) within the $5 \mathrm{G}$ core network [6], to map the traffic to appropriate Xcast QoS flows within each slice. The QoS flows are mapped to Xcast data radio bearers, using the same principles as in the case of the transport network to determine the appropriate mode of over-the-air transmission. The main idea here is to enable vertical and OTT service providers to deliver traffic with controllable and configurable quality of experience. Similarly, in MATILDA the slice intent will be translated and mapped to the most relevant network slice template advertised by a network operator, considering the set of denoted QoS requirements.
In 5G-TRANSFORMER, the Vertical Slicer (VS) is a common entry point for all vertical industries. It coordinates and arbitrates vertical slice requests for the use of networking and computing resources. Slices are requested at the VS through a new defined interface using templates with simple interconnection models, thus relieving the vertical industry from specifying its slice details. A template including the information provided by a vertical is called Vertical Service Descriptor (VSD) and can be either based on basic components and interfaces to compose the service, yielding to a service graph similar to a forwarding graph from NFV, or be based on a set of essential services used as building blocks to compose more complex services. The VS is therefore in charge of mapping the high-level requirements and placement constraints of the slice template into a set of one or more VNF/VA graphs and service function chains (SFCs).

In BlueSPACE, multi-tenancy and network slicing are adopted to improve the efficiency in the utilization of fronthaul resources. BlueSPACE will develop a Network Slice Manager (NSM), working on top of the NFVO and responsible for the lifecycle management of the network slices, following the 3GPP definition and modelling of network slices. The NSM is in charge of managing the lifecycle of network slice instances and translates their QoS requirements into suitable NFV network services which are instantiated and scaled on-demand at the NFVO, based on the evolution of the associated slice.

With regards to slice elasticity aspects, it is the main focus of the 5G-MonArch project, through the introduction of a set of scalable slice resource scheduling mechanisms, enabling the network to adapt to fluctuating network loads without requiring extreme resource overprovisioning.

Another set of approaches target also the slices creation for specific domains. To address capacity limitations and highspeed mobility requirements of future railway systems, in 5GPICTURE, a flexible control plane offering the ability to create infrastructure slices over the heterogeneous network platform is detailed. Through this approach, railway system operators will be able to instantiate and operate several virtual infrastructures enabling multi-tenancy, supporting jointly energy and telecom services. 5GCity, focusing on Smart Cities solutions, actuates the network slicing mechanisms through the Neutral Host model, which consists in managing a network infrastructure to host (without imposing technical and economic constraints) any entity that uses it to provide its services to its end users. In this model, the "Neutral Host", i.e. the infrastructure owner, is able to operate a partition of its resources and to arrange them in a set of homogeneous tenants (or slices) and expose them to a service provider which in turn uses these resources to compose the portfolio of its services. 5GCAR, targets automotive safety and infotainment improvements by developing an overall 5G V2X system architecture including components for slice management in the automotive domain. Finally, the IoRL Architecture allows the home owner or building services manager to have connectivity to different mobile network operators and to slice the building radio-light network resources between them to facilitate the use of different devices registered with different operators, as well as exploiting the license-free VLC and mmWave spectrum for accessing the home network. 


\section{MANAGEMENT AND ORCHESTRATION}

As already mentioned in the existing challenges for management and orchestration aspects, there is a need for interoperability and separation of concerns among the orchestration mechanisms across various layers (applications /MEC orchestration, NFV/SDN orchestration, OSS/BSS systems and network slices management). In this section, a short reference to existing solutions provided by 5G PPP projects is presented, aiming at categorizing the set of solutions along with the main orchestration layers that they tackle.

MATILDA aims to provide an end to end orchestration solution tackling aspects related to orchestration of the vertical applications along with orchestration of the set of network services activated within the created network slices. However, focus is given mainly on the vertical applications orchestration and its interoperability with existing OSS/BSS systems and NFV orchestrators. The main objective is to manage the optimal deployment and execution of vertical applications over the 5G network slices incorporating intelligence through various mechanisms. 5G-TRANSFORMER proposes an open and flexible transport and computing platform at the "5G Edge" tailored for verticals. It leverages on the concept of network slicing and virtualization together with native SDN and NFV control to flexibly distribute VNFs in MEC and Cloud platforms. Abstraction levels allow "as a service" resources provisioning for verticals' applications; slicing creation with required network functions and SLA; and processed/filtered monitoring features. In order to build such an architecture, it aims to extend the ETSI MANO design adding new building blocks, namely the Vertical Slicer and the Service Orchestrator interworking with the Mobile Transport Platform defined in the 5G-Crosshaul project [2]. In both MATILDA and 5G-TRANSFORMER, use cases stemming from the MEC and Cloud Computing areas are representative of the main considered applications.

The 5GTANGO Service Platform (SP) regards an open source MANO framework that provides the service and function orchestration features, plus all the needed complementary and supporting features, like slice management, policy and SLA management, user access management and infrastructure adapter. SP's clients are the OSSs/BSSs of the platform owner, as well as the (also from 5GTANGO) SDK and the Validation and Verification (V\&V) platform that are targeted to development, validation and verification of NSs. 5GTANGO SP is ETSI NFV compliant and thus able to support all the NFV oriented use cases.

Through adopting the ETSI NFV MANO architecture, 5GCity is building a framework which will provide different mechanisms in addition to lifecycle management, in order to enable both data-centric programming model for edge-based services, optimized resource consumption at edge and micro datacenters (through unikernels) and a SDK towards thirdparty users who will be enabled to dynamically deploy their own services over the distributed edge city infrastructure. Through the modular virtualization capabilities activated and coordinated by the 5GCity orchestrator layer, it is possible to monitor and manage multiple performance metrics of the $5 \mathrm{G}$ virtualized infrastructure. These performance metrics can be used to better differentiate the network capabilities, feed in the decision engines for resource allocation and optimization, and guarantee QoS/QoE targets to individual service requests.

In the context of the 5G-MEDIA project, the 5G-MEDIA SDK interacts with the Service Virtualization Platform (5GMEDIA SVP), which hosts the components related to the ETSI MANO framework (NFV Orchestrator, VNF Manager(s), Infrastructure Manager(s) and Virtualization \& Abstraction Layer), the 5G-MEDIA NSD/VNFD Catalogue as well as generic components that can be used by many applications (e.g. monitoring and optimization components). A specific innovation of the 5G-MEDIA project is the integration of the Cognitive Network Optimizer (CNO) within the SVP as part of the Media Service MAPE (MonitoringAnalyse-Plan-Execute) component. The CNO comprises mechanisms that take advantage of machine learning techniques and optimization policies management and will trigger the dynamic instantiation of VNF Forwarding Graphs on the different NFVIs. The Cognitive Network Optimizer is able to respond to dynamic changes of the environment and to adapt the deployment of VNF forwarding graphs seamlessly to continuously meet expected QoS requirements.

In the IoRL project, orchestration is based on the adoption and extension of an NFV Orchestrator (NFVO). The NFVO receives appropriate commands from the upper layer (i.e. application layer) by use-case specific applications, which include the Logic of each use-case and provide to the NFVO appropriate NS descriptors, which initiates the VNF instantiation with the appropriate network configuration.

The BlueSPACE project extends the ETSI NFV MANO architecture to meet the specific transport technologies and functionalities considered in the project. The proposed SDM/ WDM-enabled fronthaul network will be integrated with the NFVO, since currently the NFVO-WIM interface is not widely implemented and still lacking maturity. BlueSPACE will study the integration of MEC applications with NFV aiming at sharing the NFVI-PoPs and the NFV MANO stack. BlueSPACE will also develop specific PNF managers, as well as the agents that performs the actual configuration of the PNFs. Finally, BlueSPACE will also extend NFVO and WIM to enable the computation and provisioning of strict network paths and cloud resource allocation algorithms by the NFVO.

\section{CARTOGRAPHY OF 5G VERTICAL ENABLERS}

The enabling technologies presented in the previous sections represent a unique set of design and development assets, validation and verification kits, resources and slice management and orchestration mechanisms that can significantly impact on the advent of $5 \mathrm{G}$ in production networks for innovative services offered to the Verticals. These research and innovation elements are part of coherent strategy that is being realized by various $R \& D$ projects of the $5 \mathrm{G}$ PPP programme. A visual cartography of the main research aspects tackled by the projects presented in this paper is provided in Figure 1, showing their main research focus. It should be noted that the set of presented enablers does not represent an exhaustive list of the full 5G PPP research and innovation portfolio. 


\section{CONCLUSIONS}

Telecommunication and vertical industries are coming together to enable improved solutions based on the evolving $5 \mathrm{G}$ technology. The network needs to be very flexible to meet the wide range of challenges that the different verticals are facing. Various solutions, such as slice management, edge computing, network functions virtualization, and overall orchestration are brought forward as mechanisms to enable this flexible network. In the current manuscript, upon short presentation of the challenges faced by verticals for exploiting

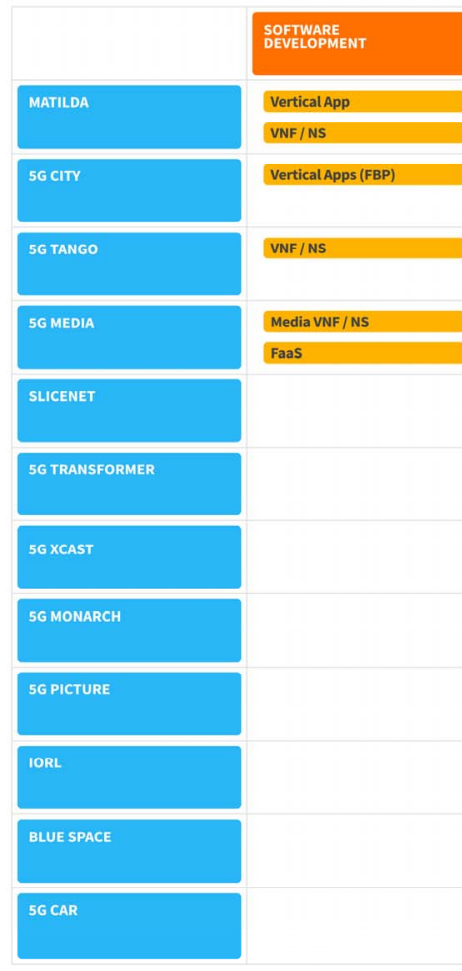

5G technologies, a cartography of the existing solutions under development in various $5 \mathrm{G}$ PPP projects is provided. Complementary solutions are designed covering the overall lifecycle of vertical applications development, formal description of network requirements and translation to the creation of network slices, management of the required network services per vertical domain, as well as orchestration of a set of mechanisms. Based on the cartography, synergies among projects can be examined leading to enhancing the overall interoperability of the provided solutions.
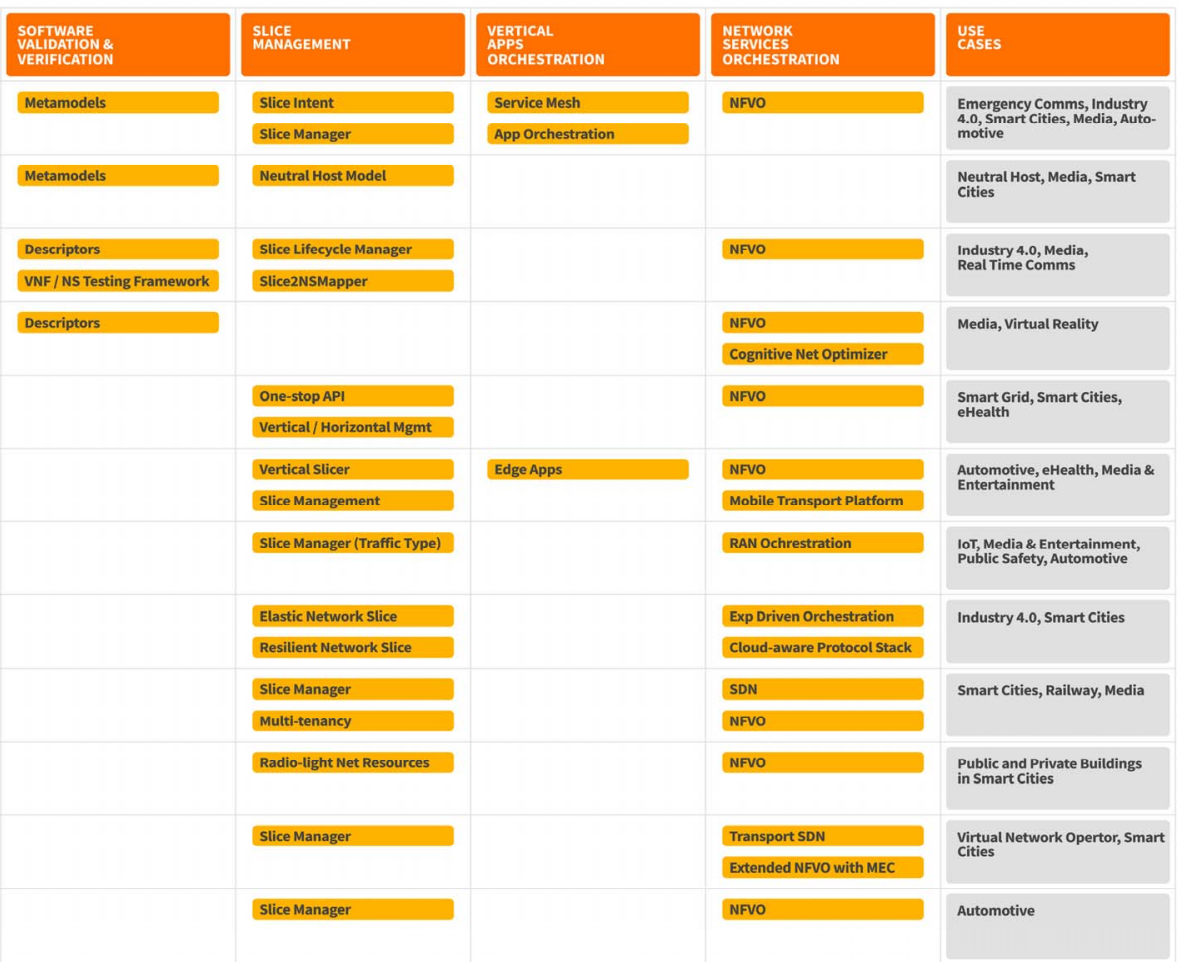

Figure 1: Cartography of 5G Vertical Enablers

\section{ACKNOWLEDGMENT}

This work was supported by the European Commission through the 5G PPP Projects MATILDA (GA No. 761898), 5GTANGO (GA No. 761493), 5GCITY (GA No. 761508), 5GMEDIA (GA No. 761699), SLICENET (GA No. 761913), 5G-TRANSFORMER (GA No. 761536), 5G-Xcast (GA No. 761498), IoRL (GA No. 761992), 5G-PICTURE (GA No. 762057), 5G-MoNArch (GA No. 761445), 5GCAR (GA No. 761510), BlueSPACE (GA No. 762055).

\section{REFERENCES}

[1] 5G PPP Architecture Working Group , 5G empowering vertical industries, February 2016, Online: https://5g-ppp.eu/wpcontent/uploads/2016/02/BROCHURE 5PPP BAT2 PL.pdf

[2] 5G Vision, The 5G Infrastructure Public Private Partnership: the next generation of communication networks and services, Online: https://5gppp.eu/wp-content/uploads/2015/02/5G-Vision-Brochure-v1.pdf
[3] 5G PPP Architecture Working Group, View on 5G Architecture, Online: https://5g-ppp.eu/wp-content/uploads/2014/02/5G-PPP-5G-ArchitectureWP-For-public-consultation.pdf

[4] ETSI Network Function Virtualization (NFV), Architectural Framework, Online: http://www.etsi.org/deliver/etsi_gs/nfv/001_099/002/01.01.01_60/gs_nf v002v010101p.pdf

[5] NGMN Alliance, Description of Network Slicing Concept, January 2016

Online: https://www.ngmn.org/uploads/media/160113 Network_Slicing_v1 0.p df

[6] 3GPP TS 23.501, "System Architecture for the 5G System," Dec. 2017.

[7] A. Prasad, M.A. Uusitalo, D. Navratil, and M. Säily, "Challenges for Enabling Virtual Reality Broadcast Using 5G Small Cell Network," IEEE WCNC Workshops (WCNCW), Barcelona, April 2018.

[8] Morrison, J. P. (1994). Flow-based programming. In Proc. 1st International Workshop on Software Engineering for Parallel and Distributed Systems (pp. 25-29)

[9] K. Samdanis, X. Costa-Perez, V. Sciancalepore, "From Network Sharing to Multi-tenancy: The 5G Network Slice Broker," IEEE Communications Magazine, 54(7), pp. 32-39, 2016.

[10] H. Zhang, et al., "Network Slicing Based 5G And Future Mobile Networks: Mobility, Resource Management, and Challenges," IEEE Communications Magazine, vol. 55(8), pp. 138-145, 2017 